\title{
The Role of the Plasma Current in Turbulence Decrease with Lower Hybrid Waves
}

\author{
Ghassan Antar ${ }^{1, *}$, Marc Goniche $^{2}$, and Annika Ekedahl ${ }^{2}$ \\ ${ }^{1}$ American University of Beirut, Riad el-Solh, Beirut 1107-2020, Lebanon \\ ${ }^{2}$ CEA, IRFM, F-13108 Saint Paul-lez-Durance, France
}

\begin{abstract}
We study the effect of lower hybrid waves on edge turbulence using a set of two Langmuir probes inserted in the scrape-off layer of the Tore Supra tokamak. We use the cross-correlation coefficient to assess the interplay between turbulence and the LH waves. We found that turbulence is affected at high plasma current even at relatively low LH powers. When the plasma current is below 1 MA, this effect disappears. Finally, we show that the level of fluctuations and the cross-correlation amplitude are correlated whereas the increase of the first leads to the increase of the second. The role of the plasma current could reflect that the exchange of the RF waves with edge turbulence is occurring in the closed field lines rather in the open field lines range.
\end{abstract}

\section{Introduction}

The interaction between the radio frequency (RF) waves and the edge turbulence has resurfaced recently after experimental results showing that turbulence in the SOL decreases when the ion cyclotron frequency heating (ICRH) is switched on [1,2]. Using GHz-range lower hybrid (LH) waves to drive current into tokamak plasmas, this issue presented mixed results. Some tokamaks (FTU \& HT-7) have shown a net decrease of turbulence $[3,4]$ while others (Tore Supra) did not $[5,6]$. In a recent paper, we showed that these apparent discrepancies among tokamaks and RF wave frequencies were removed where it is found that turbulence largescale structures in the scrape-off layer decrease at high enough plasma currents $\left(\mathrm{I}_{\mathrm{p}}\right)$ on the Tore Supra tokamak [7]. We distinguished three regimes: At low $\mathrm{I}_{\mathrm{p}}\left(\mathrm{I}_{\mathrm{p}}<0.7\right.$ MA) no modification is detected with statistical properties of turbulence similar to ohmic plasmas even with a lower hybrid power $\mathrm{P}_{\mathrm{LH}}$ reaching $4.8 \mathrm{MW}$. Moderate plasma currents $\quad\left(1.2>\mathrm{I}_{\mathrm{p}}>0.7 \mathrm{MA}\right)$, turbulence is modified at high $\mathrm{LH}$ power. At high plasma currents $\left(I_{p}>1.2 \mathrm{MA}\right)$, a net decrease in the level of turbulence of about $30 \%$ even with moderate LH power is recorded. The level of turbulence is defined as the standard deviation of the signal normalized by its average value.

The main goal of this paper is to show that at turbulence large-scale structures decrease when both the lower hybrid power and the plasma current are moderately high. Then, we shall correlate the level of turbulence to the amplitude of the cross-correlation coefficient and deduce that at both the plasma current plays an important role in the interaction between the RF waves and edge turbulence. This leads to a change in the confinement properties as we show by comparing the dependence of the diamagnetic energy on the plasma current at low vs high RF power.

\section{Plasma Scenarios}

The experiments studied here are performed on the Tore Supra tokamak. The plasma current as well as the LH power are varied while keeping the same toroidal magnetic field, $\mathrm{B}_{\phi}=3.82 \mathrm{~T}$. The major and minor radii are constant set to 2.4 and $0.7 \mathrm{~m}$ respectively and we verified that the distance between the last closed flux surface (LCFS) and the probes is constant as this could modify the level of fluctuations. The LH power is delivered by two multi-junction LH launchers $\mathrm{C} 3$ and $\mathrm{C} 4$ with a peak of the $\mathrm{N}_{\| 0}$ spectrum launched at 1.82. The power is distributed almost equally between the two launchers. The two probes used are in the $\mathrm{C} 4$ launcher and a constant bias of $-100 \mathrm{~V}$ is applied, leading to signals reflecting the ion saturation current $\mathrm{I}_{\text {sat. }}$. They are flush-mounted at the mid-plane with a diameter of $5 \mathrm{~mm}$ and a distance of $1 \mathrm{~cm}$ between them. They are positioned at 3.158 and $3.164 \mathrm{~m}$ from the torus axis for the shots $47654-47666$ and $47744-47757$ respectively, the LCFS being at $3.09 \mathrm{~m}$ and the plasma decay length is $2-3 \mathrm{~cm}$. The launcher front face and thus the probes are protected by side limiters protruding by $2 \mathrm{~mm}$. The field lines in front of the probes are always magnetically connected to the active lower hybrid launchers but not at the same place. The field lines passing in front of the probes are always magnetically connected to the upperhalf of $\mathrm{C} 3$. With respect to $\mathrm{C} 4$, they are connected to the bottom of few waveguides at low plasma currents $\left(I_{p} \sim\right.$ 0.7 MA) whereas they are connected to most of the

Corresponding author: ghassan.antar@aub.edu.lb 
waveguides at high plasma current ( $\sim 1.2 \mathrm{MA})$ (see Fig. 1). The signals studied here are sampled with DFLUC at $1 \mathrm{MHz}$, with 13,600 points per trigger per channel.

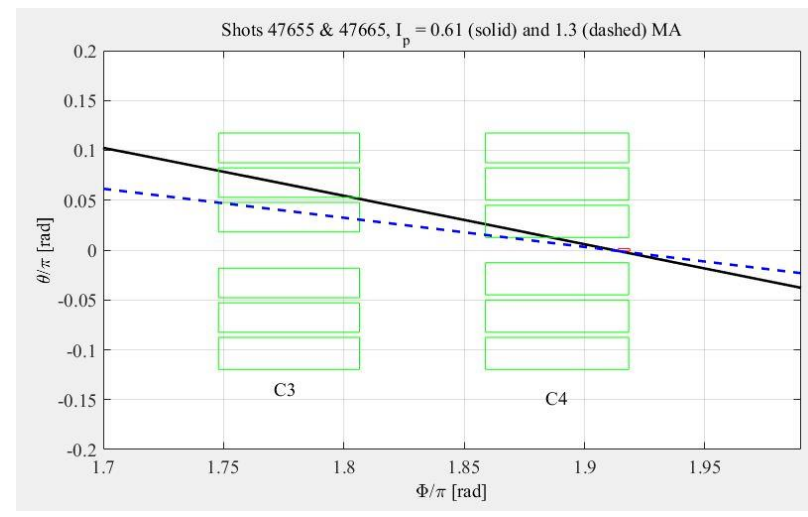

Fig. 1. The magnetic field lines in front of the probes. The magnetic field is $3.77 \mathrm{~T}$ with plasma minor and major radii equal respectively to 0.72 amd $2.38 \mathrm{~m}$.

\section{The Cross-correlation Coefficient Behavior}

The effect of the plasma current and power on the fluctuations in the SOL is measured by the two Langmuir probes. We use the cross-correlation coefficient defined as

$$
C_{12}(\tau)=\frac{\left\langle I_{1}(t) I_{2}(t+\tau)\right\rangle}{\left\langle I_{1}^{2}\right\rangle^{1 / 3}\left\langle I_{2}^{2}\right\rangle^{1 / 3}}
$$

where $I_{1}\left(I_{2}\right)$ is deduced from the ion saturation current of probe 1(2) after subtracting the average value leading to $\mathrm{I}_{1}=\mathrm{I}_{\text {sat }, 1}-\left\langle\mathrm{I}_{\mathrm{sat}, 1}\right\rangle$. We plot the cross-correlation coefficient $\mathrm{C}_{12}$ as a function of time for five cases in Fig. 2. Data is recorded before the discharge shows that data are not correlated at all between probe 1 and 2 . During ohmic discharge, the amplitude of $\mathrm{C}_{12}$ reaches 0.8 and a width about $100 \mu \mathrm{s}$. The width of $\mathrm{C}_{12}$ describes the duration of an event. It decreases from about 100 to $10 \mu$ s with increasing $\mathrm{I}_{\mathrm{p}}$ with $\mathrm{P}_{\mathrm{LH}}=4.6 \mathrm{MW}$. This could be caused by, (1), an increase in the poloidal and/or toroidal convection velocity leading to structures moving faster by the probe, (2), the decrease of the large-scale structures or (3) a combination of the two. Consequently, with the width of $\mathrm{C}_{12}$ we are unable to distinguish among the three possibilities. The amplitude of the crosscorrelation coefficient $\left(\operatorname{Max}\left(\mathrm{C}_{12}\right)\right)$ on the other hand is dominated by structures with length scales greater or equal than the separation between the probes, that is, about $1 \mathrm{~cm}$. It is not directly affected by the convective velocity in the poloidal or toroidal directions. For $\mathrm{P}_{\mathrm{LH}}=4.6 \mathrm{MW}$ one can see that the amplitude drops from 0.65 to 0.38 indicating a net decrease in the structures which have length scales greater than $1 \mathrm{~cm}$; This is not the case when increasing the LHCD power from 0 to 2.2 MW. We deduce that at least part of the width decrease is caused by a net decrease in the turbulent structures' length scales. Hereafter, we shall use $\operatorname{Max}\left(\mathrm{C}_{12}\right)$ to assess the existence of large scales in the turbulent plasma since, unlike the width, it is independent of the velocity of the structures.

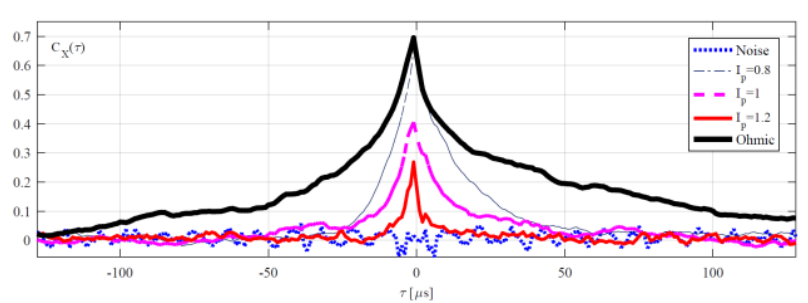

Fig. 2. The dependence on the time lag $\tau$ of $\mathrm{C}_{12}$ under different conditions: no plasma reflecing instrumental noise (dotted line), ohmic plasma (thick solid), LH current drive plasmas with different plasma currents. Note that only at high plasma currents that the amplitude of the cross-correlation decreases whereas the width, which could be dominated by the convection velocity decreases as soon as $\mathrm{P}_{\mathrm{LH}}$ is turned on.

\section{The Cross-Correlation Amplitude as a Function of the LH Power and Plasma Current}

We determine $\operatorname{Max}\left(\mathrm{C}_{12}\right)$ for a variety of discharges and the result is shown in Fig. 3. The plasma discharges have in common most of the plasma parameters such as the main magnetic field and most importantly the distance between the probe and the last closed flux surface. On the other hand, they have different plasma currents and LH powers; The plasma density was not the same but we have shown that the turbulence parameters are not modified with increasing density [7].

As a function of the plasma current, the subplot in Fig. 3(a) shows that for $I_{p}<1 M A, \operatorname{Max}\left(C_{12}\right)$ is almost unchanged and is about 0.8 . However, one can note that for Ip $\sim 1 \mathrm{MA}$, a couple of points lie around 0.6. At higher plasma currents, $\mathrm{I}_{\mathrm{p}}>1.2$, most of the recorded amplitudes are below 0.6 reaching 0.4 for $\mathrm{I}_{\mathrm{p}}>1.4 \mathrm{MA}$. This figure clearly shows that the amplitude of the crosscorrelation coefficient decreases in the presence of $\mathrm{LH}$ waves but this behavior appears to depend on $\mathrm{I}_{\mathrm{p}}$.

As a function of the $\mathrm{LH}$ power, we plot $\operatorname{Max}\left(\mathrm{C}_{12}\right)$ in subplot Fig. 3(b). For low $\mathrm{P}_{\mathrm{LH}}$ most of the amplitudes are about 0.8 with the exception of two data points for $\mathrm{P}_{\mathrm{LH}}$ equals to 2.5 and $3 \mathrm{MW}$. For high power values, the error around $\operatorname{Max}\left(\mathrm{C}_{12}\right)$ becomes large with values varying between 0.8 , the low power estimate, to 0.4 . When putting the two dependencies together, it appears that in order to obtain a smooth transition, one must have high current and high LH power. The same data points are now plotted as a function of $\mathrm{I}_{\mathrm{p}} \mathrm{P}_{\mathrm{LH}}$ in order to highlight this fact in Fig. 3(c). Clearly now when the product is below $3 \times 10^{12} \mathrm{WA}$, no dependence is reported whereas when it is greater a net decrease is observed and is proportional to the product. More theoretical work is needed to understand this dependence. 

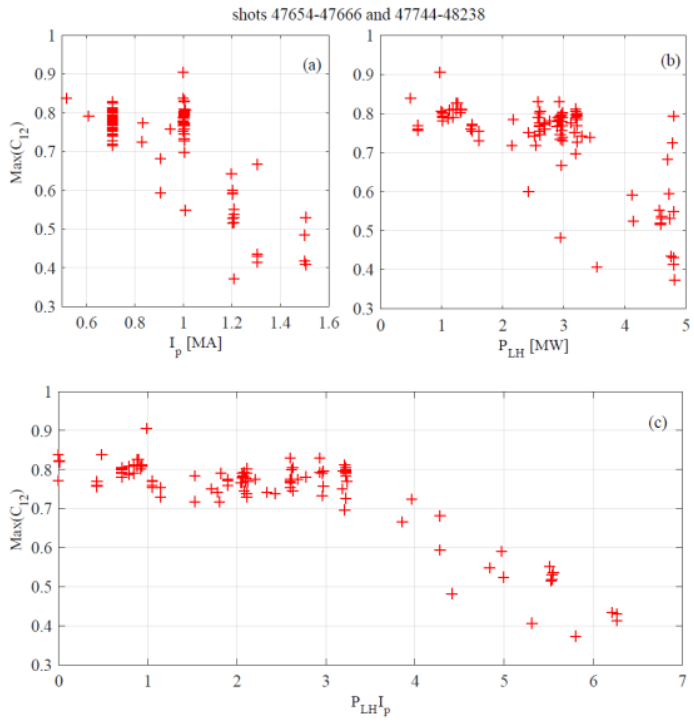

Fig. 3. (a) the cross-correlation amplitude as a function of the plasma current, (b) the same but with respect to the LH power, and (c) the same data points plotted against the product of the LH power by the plasma current.

\section{Relationship between The Cross- Correlation Amplitude and the Level of Fluctuations}

Turbulence transport is mainly driven by the large-scale structures which dominate its mixing properties [10]. When plasma is transported outside the last closed flux surface, it becomes connected to the solid objects, either a limiter or a divertor. Consequently, and because of the parallel motion, the small-scale structures are mostly affected leading to the decrease of their presence in the scrape-off layer. For the above results, we used the amplitude of the cross-correlation coefficient which is directly linked to the size the turbulent structures. In Fig. 4, $\operatorname{Max}\left(\mathrm{C}_{12}\right)$ is plotted against the level of fluctuations, defined as the standard deviation normalized by the average value of the signals, denoted by $\delta I / I$. One can deduce that higher correlation amplitudes lead to a larger level of fluctuations confirming the above hypothesis and allowing us to link the effects of the plasma current and LH power to the level of fluctuations

\section{Level of Fluctuations and confinement}

At a constant LH power, Fig. 5 shows the behavior of the level of fluctuations with respect to the plasma current. It decreases with increasing $I_{p}$ in agreement with the decrease of $\operatorname{Max}\left(\mathrm{C}_{12}\right)$. We approximated this dependence by a power law of the type $\mathrm{I}_{\mathrm{p}}^{-1 / 2}$ in reference to earlier results on the Tore Supra tokamak [8].

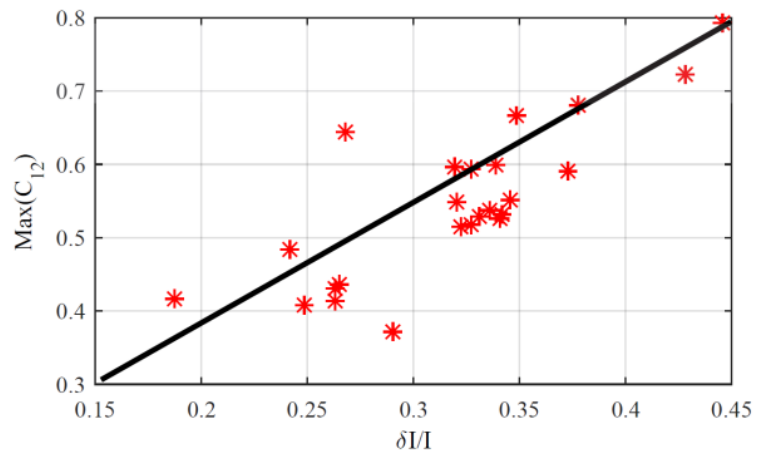

Fig. 4. The maximum of the cross-correlation coefficient is plotted against the level of fluctuations $(\delta I / I)$ where the increase of the second is correlated to the increase of the first.

In squares the energy content of the plasma increases with $I_{p}$. The same behavior is obtained after the RebutLallia-Watkins (RLW) scaling is applied to the discharge parameters. Meanwhile, the level of turbulence decreases with $I_{p}$. This could reflect the link between global confinement properties, the plasma current and SOL turtbulence.

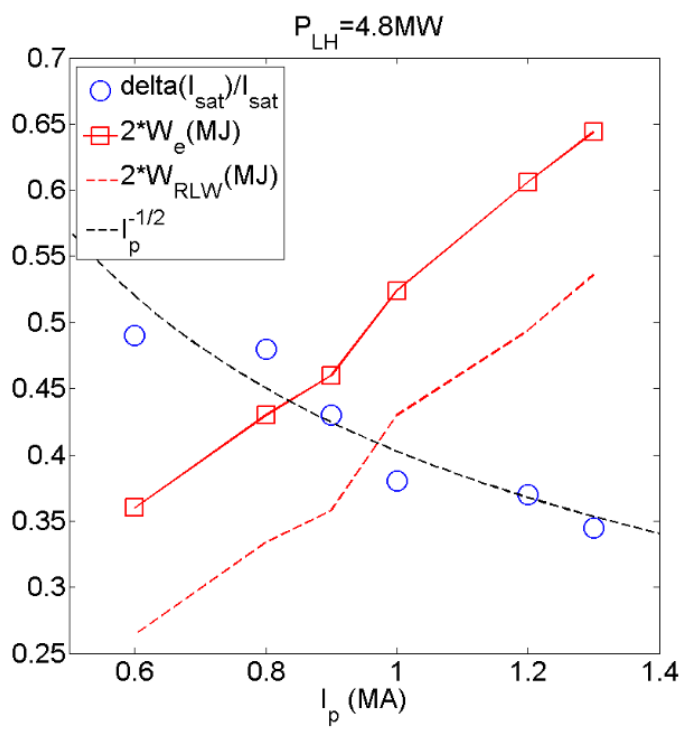

Fig. 5. The level of fluctuations In (o) as a function of the plasma current fitted by $\mathrm{I}_{\mathrm{p}}^{-1 / 2}$, in squares the total electron energy and In dashed line the RLW scaling.

Consequently, for these discharges the confinement of the electrons (no ion heating) follows the general trend observed on Tore Supra for LHCD discharges [9]. At $\mathrm{P}_{\mathrm{LH}}=400 \mathrm{~kW}$, the electron stored energy follows the Rebut-Lallia-Watkins scaling and at high power $\left(\mathrm{P}_{\mathrm{LH}} \sim 4.8 \mathrm{MW}\right)$, it is above the RLW scaling by a factor increasing when $\mathrm{I}_{\mathrm{p}}$ is decreasing. This could be the result of the internal transport barrier being built when increasing fraction of current is driven by LH waves; Further analysis of the plasma profiles is needed to support this hypothesis. So, at high power we find a good correlation between the fluctuation rate measured near the LHCD antenna and the confinement when $\mathrm{I}_{\mathrm{p}}$ is changed. At low power, we varied $\mathrm{I}_{\mathrm{p}}$ by more than $70 \%$ going from $0.8 \mathrm{MA}$ to $1.3 \mathrm{MA}$ and the fluctuations rate 
was found to be almost unchanged. The behavior of the level of fluctuations with respect to $I_{p}$ is depicted in Fig. 6 for low and high power RF waves. The two behaviors as a function of the current can be clearly distringuished because of the LH power.

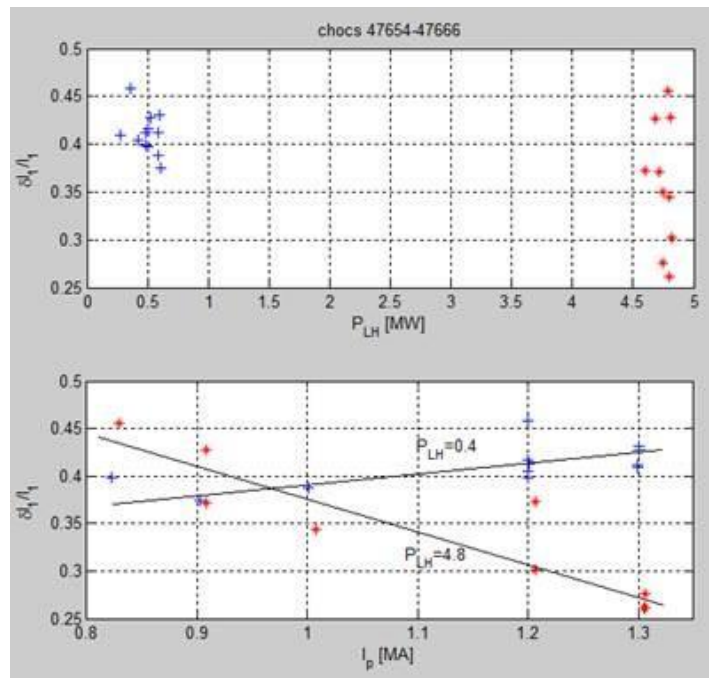

Fig. 6. The level of fluctuations sa a function of $I_{p}$ for the low $\mathrm{RF}$ power data $(+)$ and for the high RF power $\left({ }^{*}\right)$.

\section{Conclusion}

In agreement with our previous publications, the present results show the important role of the plasma current in the way the LH power affects edge turbulence. We use the amplitude of the cross-correlation coefficient between two Langmuir probes positioned in the SOL and toroidally distanced by $1 \mathrm{~cm}$. Several conclusions can be drawn from this work:

1- We confirm that the apparent discrepancy among tokamaks is lifted where turbulence decrease due to RF waves is now observed on all tokamaks where it has been studied.

2- We also confirm that the apparent descripency between RF waves is also lifted. RF waves around 50 $\mathrm{MHz}$ are used for plasma heating and those around 1 $\mathrm{GHz}$ for current drive. They both are capable of affecting turbulence in the SOL and in quite a similar fashion.

3- Clearly, the plasma currrent plays an important role in the way the RF power at the $\mathrm{GHz}$ frequencies affect turbulence. This opens up the question about the interplay between the edge where some power is deposited and the SOL where the measurements are made.

4- The effect of magnetic connection may not be important since the field lines in front of the probes are always connected to the active coupler. This is supported by the fact that turbulence decreases at a constant $I_{p}$ but with increasing LH power. In this case the magnetic field lines are unmodified but the LH power is.

For future work, we will continue studying the issue of the threshold and the role of the plasma current. We will be comparing the effects of the RF waves at the
ICRH frequencies to those at the $\mathrm{LH}$ frequencies on the turbulence.

\section{References}

[1] Antar, G., et al. "Convective transport suppression in the scrape-off layer using ion cyclotron resonance heating on the ASDEX Upgrade tokamak." Physical review letters 105.16 (2010).

[2] Antar, G. Y., et al. "The role of power and magnetic connection to the active antenna in the suppression of intermittent structures by ion cyclotron resonance heating." Nuclear Fusion 52.10 (2012): 103005.

[3] Ding, B. J., et al. "Effect of MHD on edge electrostatic fluctuation with lower hybrid current drive in the HT-7 tokamak." Plasma physics and controlled fusion 46.9 (2004): 1467.

[4] Ridolfini, V. Pericoli, et al. "Lower hybrid current drive efficiency in tokamaks and wave scattering by density fluctuations at the plasma edge." Nuclear Fusion 51.11 (2011): 113023.

[5] T Oosako et al., In RADIO FREQUENCY POWER IN PLASMAS: Proceedings of the 19th Topical Conference, volume 1406, 239 (2011).

[6] Goniche, M., et al. "Lower hybrid current drive at high density on Tore Supra." Nuclear Fusion 53.3 (2013): 033010.

[7] Antar, G., et al. "The role of the plasma current in turbulence decrease during lower hybrid current drive." Physics of Plasmas 24.3 (2017): 032307.

[8] Vermare, L., et al. "Density fluctuation measurements using X-mode fast sweep reflectometry on Tore Supra." Nuclear fusion 46.9 (2006): S743.

[9] Hoang, G. T., et al. "Improved confinement in high li lower hybrid driven steady state plasmas in Tore Supra." Nuclear fusion 34.1 (1994): 75.

[10] Diamond, P. H., S.-I. Itoh, and K. Itoh. Modern Plasma Physics: Volume 1, Physical Kinetics of Turbulent Plasmas. Cambridge University Press, 2010. 